Pacific Journal of Mathematics

INTERSECTIONS OF THE SPACE OF SKEW-SYMMETRIC
MAPS WITH ITS TRANSLATES 


\title{
INTERSECTIONS OF THE SPACE OF SKEW-SYMMETRIC MAPS WITH ITS TRANSLATES
}

\author{
DANIEL B. Shapiro
}

For a quadratic space $V$ over a field $K$, let $\mathscr{L} \subseteq \operatorname{End}(V)$ be the space of all maps which are skew-symmetric with respect to the inner product. For $g \in \mathrm{GL}(V)$, let $\mathscr{D}(g)=$ $\operatorname{dim}(\mathscr{L} \cap g \mathscr{L})$. In this paper we determine the largest few values possible for $\mathscr{D}(g)$, and we classify the maps $g$ which achieve these values. The restriction of this result to maps $g$ in the orthogonal group $O(V)$ generalizes the characterization of \pm symmetries originally proved by Botta and Pierce.

1. Introduction. Let $K$ be a field of characteristic not two and let $(V, B)$ be a quadratic space of dimension $n$ over $K$. That is, $V$ is a $K$-vector space of dimension $n$ and $B: V \times V \rightarrow K$ is a non-degenerate symmetric bilinear form. Let $G=\mathcal{O}(V, B)$ be the orthogonal group of this space. Let $\mathscr{L}$ be the space of all elements of End $(V)$ which are skew-symmetric with respect to $B$. For $g \in G$, the space $g \mathscr{L}=$ $\{g \circ h \mid h \in \mathscr{L}\}$ may be considered as the tangent space to $G$ at the point $g$.

For $g \in \operatorname{End}(V)$, let

$$
\mathscr{D}(g):=\operatorname{dim}(\mathscr{L} \cap g \mathscr{L}) .
$$

This dimension $\mathscr{D}(g)$ is viewed as a measure of how far $g$ is from being a scalar map. For $a \in K^{\times}, \mathscr{D}(a 1)=\operatorname{dim} \mathscr{L}=\frac{1}{2} n(n-1)$. The following theorem, due to Botta and Pierce, shows that the non-scalar elements $g \in G$ which have the largest value for $\mathscr{D}(g)$ are the \pm symmetries. For an anisotropic $v \in V$, the symmetry (or hyperplane reflection) corresponding to the line $K v$ is the map $\tau_{v} \in G$ which sends $v$ to $-v$ and which pointwise fixes the hyperplane $(K v)^{\perp}$. Then

Theorem 1.1. [1, Prop. 7.1.]. Suppose $n \geqq 3$ and $g \in G, g \neq \pm 1$.

$$
\mathscr{D}(g) \leqq \frac{(n-1)(n-2)}{2}
$$

with equality holding if and only if $\pm g$ is a symmetry.

The purpose of the present paper is to determine the next largest values for $\mathscr{D}(g)$, and to determine which maps $g$ attain these 
values. The value of $\mathscr{D}(g)$ is computed in terms of the Jordan form of $g$, by interpreting $\mathscr{D}(g)$ as the dimension of the kernel of the linear map $g \otimes 1-1 \otimes g$ on $\Lambda^{2} V$. The Jordan forms of all $g \in G L(V)$ having $\mathscr{D}(g) \geqq \frac{1}{2}(n-2)(n-3)$ are found, using some manipulations with partitions. Then, we utilize results of Milnor [2] to restrict attention back to elements $g$ in the orthogonal group. The final result is stated below.

We assume $K$ is algebraically closed here, but this is not much loss of generality (see Remark 1.5). The equalities stated in the theorem mean that, with respect to some basis of $V$, the matrix of $g$ equals the indicaled matrix. The symbol, $\perp$, stands for orthogonal direct sum. We write $J_{r}(1)$ for the $r \times r$ matrix in Jordan form which corresponds to the elementary divisor $(x-1)^{r}$. A subscript indicates the order of a matrix.

Theorem 1.2. Let $K$ be an algebraically closed field. Suppose $n \geqq 4$ and $g \in \mathcal{O}(V, B)$, over $K$.

(1) $\mathscr{D}(g)=\frac{n(n-1)}{2}$ iff $\pm g=1_{n}$.

(2) $\mathscr{D}(g)=\frac{(n-1)(n-2)}{2}$ iff $\pm g=$ symmetry $=1_{n-1} \perp\left(-1_{1}\right)$.

(3) $\mathscr{D}(g)=1+\frac{(n-2)(n-3)}{2}$ iff either

or

(i) $\pm g=2$-plane reflection $=1_{n-2} \perp\left(-1_{2}\right)$;

or

(ii) $\pm g=1_{n-4} \perp\left(J_{2}(1) \oplus J_{2}(1)\right)$;

(iii) $n=4$ and $g=\left(\begin{array}{cc}a 1_{2} & 0 \\ 0 & a^{-1} 1_{2}\end{array}\right)$, for some scalar $a \neq 0,1,-1$.

(4) $\mathscr{D}(g)=\frac{(n-2)(n-3)}{2}$ iff either

(i) $\pm g=1_{n-2} \perp\left(\begin{array}{cc}a & 0 \\ 0 & a^{-1}\end{array}\right)$, for some scalar $a \neq 0,1,-1$;

or

(ii) $\pm g=1_{n-3} \perp J_{3}(1)$;

or

(iii) $n=6$ and $g=1_{3} \perp\left(-1_{3}\right)$; 
(iv) $n=6$ and $g=\left(\begin{array}{cc}a 1_{3} & 0 \\ 0 & a^{-1} 1_{3}\end{array}\right)$, for some scalar $a \neq 0,1,-1$.

(5) $\mathscr{D}(g)<\frac{(n-2)(n-3)}{2}$ otherwise.

Proposition 1.3. Let $g \in G$. wise.

(1) Suppose $n=2$. Then $\mathscr{D}(g)=1$ if $g= \pm 1$ and $\mathscr{D}(g)=0$ other-

(2) Suppose $n=3$. Then $\mathscr{D}(g)=3$ if $g= \pm 1, \mathscr{D}(g)=1$ if $\pm g=$ symmetry, and $\mathscr{D}(\mathrm{g})=0$ otherwise.

REMARK 1.4. If $g$ is a product of two symmetries, then either $g$ is a 2-plane reflection, as in part (3)(i), or $g$ lies either in part (4)(i) or (4)(ii) of the theorem.

REMARK 1.5. Similar results over a general field $K$ (of characteristic not 2) quickly follow. The only parts of Theorem 1.2 that need to be changed are the forms of the special matrices in parts (3)(iii), and (4)(i), (ii), (iv). This generalization easily follows from Proposition 3.1 below, and the fact that two matrices over $K$ which are similar over the algebraic closure of $K$ must already be similar over $K$.

We would like to thank A. Wadsworth for his helpful comments which led to a substantial improvement of the theorem.

2. Computation of $\mathscr{D}(g)$. Throughout this section, we use a fixed $g \in \mathrm{GL}(V)$. We will return to the orthogonal group in Section 3.

The non-degenerate bilinear form $B$ induces an adjoint involution, $\sim$, on End $(V)$, defined by: $B(\tilde{f}(u), v)=B(u, f(v))$. If an orthonormal basis of $V$ is chosen, and matrices are used, then this involution is the transpose map.

By definition, $\mathscr{L}=\{h \in \operatorname{End}(V) \mid \tilde{h}=-h\}$. An element $h \in \mathscr{L}$ lies in $\mathscr{L} \cap g \mathscr{L}$ iff $g h=h \tilde{g}$. Let $T: \mathscr{L} \rightarrow \operatorname{End}(V)$ be defined:

$$
T(h):=g h-h \tilde{g} .
$$

Then $\mathscr{D}(g)=\operatorname{dim}(\mathscr{L} \cap g \mathscr{L})=\operatorname{dim}(\operatorname{ker} T)$.

By the usual method, the bilinear form $B$ gives an identification of $V$ with its dual space $V^{*}$, so that $\operatorname{End}(V) \cong V \otimes V^{*}$ is identified with $V \otimes V$. Furthermore, for $l, k \in \operatorname{End}(V)$, the operation $h \mapsto l h \tilde{k}$ on End $(V)$ is identified with the usual operation of $l \otimes k$ on $V \otimes V$, and the adjoint involution, $\sim$, is identified with the switch operation 
$v \otimes w \mapsto w \otimes v$ on $V \otimes V$. Therefore, $\mathscr{L} \subseteq \operatorname{End}(V)$ becomes $\Lambda^{2} V \subseteq$ $V \otimes V$, and the map $T$ above becomes the restriction to $\Lambda^{2} V$ of $g \otimes 1-1 \otimes g$.

We work with the Jordan decomposition of $V$ with respect to $g \in \mathrm{GL}(V)$. Let the elementary divisors of $g$ be $\left(x-b_{i}\right)^{l_{4}}$, for $i=$ $1, \cdots, p$. Then, $V=V_{1} \oplus \cdots \oplus V_{p}$, where $V_{1}$ is the cyclic submodule corresponding to $\left(x-b_{1}\right)^{l_{1}}$. There may be repetitions among the eigenvalues $b_{1}$; let $a_{1}, \cdots, a_{t}$ be the distinct eigenvalues. Define $V((a)):=$ the sum of all $V_{\imath}$ such that $b_{\imath}=a$, so that

$$
V((a))=\left\{v \in V \mid(g-a 1)^{k}(v)=0, \text { for some } k\right\} .
$$

Then, $V=V\left(\left(a_{1}\right)\right) \oplus \cdots \oplus V\left(\left(a_{t}\right)\right)$. Let $n_{l}:=\operatorname{dim} V\left(\left(a_{l}\right)\right)$ so that $n=$ $n_{1}+\cdots+n_{t}$. For each $j=1, \cdots, p$, the various $l_{t}$ 's corresponding to $b_{l}=a_{l}$ add up to $n_{l}$. They give a partition $P_{l}$ of the number $n_{l}$. The eigenvalues $a$, and partitions $P_{l}$ completely determine the Jordan form of $g$. We will compute $\mathscr{D}(g)$ using these partitions. The idea of using this interpretation of $\mathscr{D}(g)$ and some computations with Jordan forms is already present in [1].

Definition 2.1. Suppose $P=\left(m_{1}, \cdots, m_{k}\right)$ is a partition of $n$ into $k$ parts. That is, $n=m_{1}+\cdots+m_{k}$, where each $m_{l}$ is an integer and $1 \leqq m_{1} \leqq \cdots \leqq m_{k}$. Define

$$
\mathscr{D}(P):=\sum_{j=1}^{k}(k-j) m_{l}
$$

Proposition 2.2. For $g \in G L(V)$ as above, with partitions $P_{\imath}$ of $n_{l}$, $\mathscr{D}(g)=\mathscr{D}\left(P_{1}\right)+\cdots+\mathscr{D}\left(P_{1}\right)$.

Proof. For $v, w \in V$, define $v \wedge w:=\frac{1}{2}(v \otimes w-w \otimes v)$ and $v \circ w:=\frac{1}{2}(v \otimes w+w \otimes v)$. For subspaces $U, W \subseteq V$, define $\Lambda^{2}(U, W):=\operatorname{Span}\{u \wedge w \mid u \in U, w \in W\}$ and $S^{2}(U, W):=\operatorname{Span}\{u \circ w \mid$ $u \in U, w \in W\}$. Then, if $U \cap W=0, \operatorname{dim} \Lambda^{2}(U, W)=\operatorname{dim} S^{2}(U, W)=$ $(\operatorname{dim} U) \cdot(\operatorname{dim} W)$. If $U=W$ has dimension $l$, then $\Lambda^{2}(U):=\Lambda^{2}(U, U)$ has dimension $l(l-1) / 2$ while $S^{2}(U):=S^{2}(U, U)$ has dimension $l(l+$ 1)/2.

Since $V=V_{1} \oplus \cdots \oplus V_{p}$, we have $\Lambda^{2} V$ is the direct sum of the $\Lambda^{2}\left(V_{r}, V_{s}\right)$, for $r \leqq s$, and similarly for $S^{2} V$. Since $T(v \wedge w)=$ $g(v) \circ w-v \circ g(w)$, the map $T$ is the direct sum of the maps

$$
T_{r s}: \Lambda^{2}\left(V_{r}, V_{s}\right) \rightarrow S^{2}\left(V_{r}, V_{s}\right)
$$

for $r \leqq s . \quad$ Therefore, $\operatorname{ker} T$ is the direct sum of the $\operatorname{ker}\left(T_{r s}\right)$, for $r \leqq s$. 
Let $r \leqq s$ be fixed. Choose a basis $\left\{v_{\imath} \mid 1 \leqq i \leqq l_{r}\right\}$ of $V_{r}$ corresponding to the Jordan form; that is (setting $\left.v_{0}=0\right), \quad g\left(v_{1}\right)=$ $b_{r} v_{t}+v_{t-1}$. Choose a similar basis $\left\{w_{J} \mid 1 \leqq j \leqq l_{s}\right\}$ of $V_{s}$. It follows that

$$
T\left(v_{\imath} \wedge w_{J}\right)=\left(b_{r}-b_{s}\right) v_{l} \circ w_{l}+v_{t-1} \circ w_{l}-v_{\imath} \circ w_{l-1} \text {. }
$$

We will compute $\operatorname{dim} \operatorname{ker}\left(T_{r s}\right)$ by working with $\operatorname{coker}\left(T_{r s}\right)$, which is $S^{2}\left(V_{r}, V_{s}\right) \operatorname{modim}\left(T_{r s}\right)$. In the cases $r \neq s, \operatorname{ker}\left(T_{r s}\right)$ and coker $\left(T_{r s}\right)$ have the same dimension.

Case 1. $b_{r} \neq b_{s}$. Then, in $\operatorname{coker}\left(T_{r s}\right)$ we have $\left(b_{r}-b_{s}\right) v_{1} \circ w_{1} \equiv$ $-v_{\imath-1} \circ w_{l}+v_{\imath} \circ w_{\jmath-1}$, and an inductive argument shows that every $v_{\imath} \circ w_{\jmath} \equiv$ 0 . Hence, $\operatorname{dim} \operatorname{ker}\left(T_{r s}\right)=\operatorname{dim} \operatorname{coker}\left(T_{r s}\right)=0$.

Case 2. $\quad b_{r}=b_{s}$ but $r \neq s$. Suppose $l_{r} \leqq l_{s .}$ The space coker $\left(T_{r s}\right)$ is spanned by elements $v_{\imath} \circ w_{l}$, which satisfy the relations $v_{t-1} \circ w_{l} \equiv$ $v_{1} \circ w_{j-1}$, for $1 \leqq i \leqq l_{r}, 1 \leqq j \leqq l_{s}$. Then, every $v_{i} \circ w$, reduces either to 0 or to some $v_{k} \circ w_{l s}$. Therefore these $l_{r}$ elements span the space, so $\operatorname{dim} \operatorname{coker}\left(T_{r s}\right) \leqq l$.

Also, the space $\operatorname{im}\left(T_{r s}\right)$ is spanned by the $l_{r} l_{s}$ elements $T\left(v_{1} \wedge w_{l}\right)$. For every $k$ with $1 \leqq k \leqq l_{r}$, there is a relation $T\left(v_{k} \wedge w_{1}+v_{k-1} \wedge w_{2}+\cdots+v_{1} \wedge w_{k}\right)=0$, so that at least $l_{r}$ of these generators are redundant. Hence, $\operatorname{dimim}\left(T_{r s}\right) \leqq l_{r} l_{s}-l_{r}$, so $\operatorname{dim} \operatorname{coker}\left(T_{r s}\right)=l_{r} l_{s}-\operatorname{dimim}\left(T_{r s}\right) \geqq l_{r}$. Consequently, $\operatorname{dim} \operatorname{ker}\left(T_{r s}\right)=$ $\operatorname{dim} \operatorname{coker}\left(T_{r s}\right)=l_{r}=\min \left(l_{r}, l_{s}\right)$.

Case 3. $r=s$. Counting dimensions using the exact sequence

$$
0 \rightarrow \operatorname{ker}\left(T_{r}\right) \rightarrow \Lambda^{2}\left(V_{r}\right) \rightarrow S^{2}\left(V_{r}\right) \rightarrow \operatorname{coker}\left(T_{r}\right) \rightarrow 0,
$$

we see that $\operatorname{dim} \operatorname{ker}\left(T_{r}\right)+l_{r}=\operatorname{dim} \operatorname{coker}\left(T_{r}\right)$. The space coker $\left(T_{r}\right)$ is spanned by elements $v_{\imath} \circ v_{l}$, with relations $v_{\imath-1} \circ v_{\jmath} \equiv v_{\imath} \circ v_{\jmath-1}$, for $1 \leqq i, j \leqq$ $l_{r}$. Then, as in Case 2, every $v_{l} \circ v_{1}$, reduces either to 0 or to some $v_{k} \circ v_{l}$. Hence, at most $l_{r}$ generators are needed, so $\operatorname{dim} \operatorname{coker}\left(T_{r}\right) \leqq$ $l_{r}$. Therefore, by the equation above, $\operatorname{dim} \operatorname{ker}\left(T_{r}\right)=0$.

By Case $1, \mathscr{D}(g)$ is the sum of the $\mathscr{D}$-values of the restrictions of $g$ to the eigenspaces $V\left(\left(a_{1}\right)\right)$. For fixed $j$, suppose that $\left(x-a_{1}\right)^{m_{1}}, \cdots$, $\left(x-a_{l}\right)^{m_{k}}$ are the elementary divisors of $g$ with eigenvalue $a_{y}$, where $1 \leqq m_{1} \leqq \cdots \leqq m_{k}$. Then $P$, is the partition $\left(m_{1}, \cdots, m_{k}\right)$. By Cases 2 and 3 , the dimension of the kernel of $T$ restricted to $\Lambda^{2}\left(V\left(\left(a_{j}\right)\right)\right)$ is

$\sum_{1 \leqq r<s \leqq k} \min \left(m_{r}, m_{s}\right)=\sum_{r<s} m_{r}=(k-1) m_{1}+(k-2) m_{2}+\cdots+m_{k-1}=\mathscr{D}\left(P_{J}\right)$.

This completes the proof. 
In order to determine which partitions have large $\mathscr{D}$-values, we determine the maximal $\mathscr{D}$-value of a partition of $n$ into $k$ parts.

Definition 2.3. For integers $1 \leqq k \leqq n$, define $F_{n}(k)$ as follows:

$$
\begin{gathered}
\text { if } n=k q+r, \quad 0 \leqq r<k, \\
F_{n}(k):=\frac{k(k-1)}{2} q+\frac{r(r-1)}{2} .
\end{gathered}
$$

LEMMA 2.4. The maximal $\mathscr{D}$-value of all partitions of $n$ into $k$ parts is $F_{n}(k)$.

Proof. Suppose the partition $n=m_{1}+\cdots+m_{k}$ has the maximal $\mathscr{D}$-value. If $m_{k}-m_{1} \geqq 2$, then there exist $i<j$ such that either $m_{i}<$ $m_{l+1} \leqq m_{,-1}<m_{j}$ or $j=i+1$ and $m_{j}-m_{i} \geqq 2$. In either case, define a new partition of $n$ into $k$ parts by replacing $m_{\imath}$ by $m_{t}+1$ and $m_{j}$ by $m_{j}-1$. This new partition has larger $\mathscr{D}$-value, contrary to hypothesis. Therefore, $m_{k}-m_{1} \leqq 1$, and the partition looks like $n=$ $q+q+\cdots+q+(q+1)+\cdots+(q+1)$, for some $q$, where there are, say, $r$ of the $(q+1)$ terms $(0 \leqq r<k)$. Then $n=(k-r) q+r(q+1)=$ $k q+r$, and the (maximal) $\mathscr{D}$-value for this partition is

$$
\begin{aligned}
\mathscr{D} & =[(k-1)+\cdots+r] q+[(r-1)+\cdots+1](q+1) \\
& =\frac{k(k-1)}{2} q+\frac{r(r-1)}{2},
\end{aligned}
$$

as claimed.

REMARK. By a similar argument it follows that, for fixed $n \geqq 1$, $F_{n}(k)$ is a strictly increasing function for $1 \leqq k \leqq n$.

From the following result, we can easily find the Jordan forms of all $g \in \mathrm{GL}(V)$ with $\mathscr{D}(g) \geqq \frac{1}{2}(n-2)(n-3)$.

Proposition 2.5. Let $n=n_{1}+\cdots+n_{t}$, where $1 \leqq n_{1} \leqq \cdots \leqq n_{t}$, and let $P_{\imath}$ be a partition of $n_{1}$. Let $\mathscr{D}=\mathscr{D}\left(P_{1}\right)+\cdots+\mathscr{D}\left(P_{t}\right)$. The following list gives all cases when $\mathscr{D} \geqq \frac{1}{2}(n-2)(n-3)$.

I. $t=1$

(i) $P_{1}=(1, \cdots, 1)$ and $\mathscr{D}=\frac{1}{2} n(n-1)$.

(ii) $P_{1}=(1, \cdots, 1,2)$ and $\mathscr{D}=\frac{1}{2}(n-1)(n-2)$.

(iii) $P_{1}=(1, \cdots, 1,2,2)$ and $\mathscr{D}=1+\frac{1}{2}(n-2)(n-3)$.

(iv) $P_{1}=(1, \cdots, 1,3)$ and $\mathscr{D}=\frac{1}{2}(n-2)(n-3)$.

(v) $n=6, P_{1}=(2,2,2)$ and $\mathscr{D}=6$.

II. $t=2$

(i) $\quad P_{1}=(1), P_{2}=(1, \cdots, 1)$ and $\mathscr{D}=\frac{1}{2}(n-1)(n-2)$. 
(ii) $P_{1}=(1,1), P_{2}=(1, \cdots, 1)$ and $\mathscr{D}=1+\frac{1}{2}(n-2)(n-3)$.

(iii) $P_{1}=(1), P_{2}=(1, \cdots, 1,2)$ and $\mathscr{D}=\frac{1}{2}(n-2)(n-3)$.

(iv) $P_{1}=2, P_{2}=(1, \cdots, 1)$ and $\mathscr{D}=\frac{1}{2}(n-2)(n-3)$.

(v) $n=6, P_{1}=(1,1,1), P_{2}=(1,1,1)$ and $\mathscr{D}=6$.

III. $t=3$

(i) $P_{1}=(1), P_{2}=(1), P_{3}=(1, \cdots, 1)$ and $\mathscr{D}=\frac{1}{2}(n-2)(n-3)$.

Proof. I. $t=1$. By Proposition (2.4), $\frac{1}{2}(n-2)(n-3) \leqq \mathscr{D}\left(P_{1}\right) \leqq$ $F_{n}(k)$, where the partition $P_{1}$ has $k$ parts. If $n=k q+r, 0 \leqq r<k$, then

$$
n^{2}-5 n+6 \leqq 2 F_{n}(k)=k(k-1) q+r(r-1)=(k-1) n-(k-r) r
$$

so $n^{2}+6 \leqq(k+4) n$ and therefore $k \geqq n-3$. Suppose $P_{1}=$ $\left(m_{1}, m_{2}, \cdots, m_{k}\right)$ and $m_{1}=m_{2}=\cdots=m_{l}=1$, while $2 \leqq m_{l+1} \leqq \cdots \leqq m_{k}$. Then $n \geqq l+2(k-l)=2 k-l \geqq 2 n-6-l$, so $l \geqq n-6$. Finding all such partitions and computing their $\mathscr{D}$-values is now easily done. Note that, for any partition $P, \mathscr{D}(P) \leqq \frac{1}{2} n(n-1)$ with equality iff $P=(1, \cdots, 1)$.

II. $t=2$. By part I, $\mathscr{D} \leqq \frac{1}{2} n_{1}\left(n_{1}-1\right)+\frac{1}{2} n_{2}\left(n_{2}-1\right)$. The function $f(x)=\frac{1}{2} x(x-1)$ satisfies the following shift property: if $x \leqq y$ then $f(x)+f(y)<f(x-1)+f(y+1)$. Suppose $n_{1} \geqq 3$ (so that $n \geqq 6$ ) and shift to get $\mathscr{D} \leqq 3+\frac{1}{2}(n-3)(n-4)$. If $n>6$, this value is less than $\frac{1}{2}(n-2)(n-3)$. Then $n=6$, and since equalities hold, no shifts could have occurred. Therefore, $n_{1}=n_{2}=3$, and since $\mathscr{D}\left(P_{\imath}\right)=3$, we have $P_{1}=P_{2}=(1,1,1)$.

Suppose $n_{1}=2$. If $P_{1}=(2)$, then $\mathscr{D}=\mathscr{D}\left(P_{2}\right) \leqq \frac{1}{2}(n-2)(n-3)$, so equality must hold. Therefore, by part $I, P_{2}=(1, \cdots, 1)$. If $P_{1}=(1,1)$, then $\mathscr{D}=1+\mathscr{D}\left(P_{2}\right) \leqq 1+\frac{1}{2}(n-2)(n-3)$. If $P_{1} \neq(1, \cdots, 1)$ then, by part I, $n-2 \geqq 2$ and $\mathscr{D}\left(P_{2}\right) \leqq \frac{1}{2}(n-3)(n-4)$. Then $\frac{1}{2}(n-2)(n-3) \leqq \mathscr{D} \leqq$ $1+\frac{1}{2}(n-3)(n-4)$, and this implies $n \leqq 4$. Hence, $n=4$ and $P_{2}=$ (2). This case is already covered, after switching $P_{1}$ and $P_{2}$.

If $n_{1}=1$, then $\frac{1}{2}(n-2)(n-3) \leqq \mathscr{D}=\mathscr{D}\left(P_{2}\right)$. By part I, both $P_{2}=$ $(1, \cdots, 1)$ and $P_{2}=(1, \cdots, 1,2)$ will do, but all other cases are eliminated.

III. $t \geqq 3$. By the shift property stated in part II, we can increase $\mathscr{D}$ by cutting $n_{1}, n_{2}, \cdots, n_{t-1}$ down to 1 and increasing $n_{t}$ to $n-t+1$. Hence, $\mathscr{D} \leqq \frac{1}{2}(n-t+1)(n-t)$. If $t \geqq 4$, this value is too small. If $t=3$, equality must hold, so that no shifts could have occurred. Then $n_{1}=n_{2}=1$ and $n_{3}=n-2$. Again, since equality holds, $P_{3}$ must be $(1, \cdots, 1)$. This completes the proof.

3. Specializing to orthogonal group elements. The theorem (1.2) will follow from the results of the previous section once we know what the Jordan form of an element $g \in \mathcal{O}(V, B)$ can be. This information is found in a more general setting in [2]. We quote the relevant results. 
Let $g \in \mathcal{O}(V, B)$ be fixed. Then $V$ is a $K[x]$-module, in the usual way, and we examine the decomposition of this module into cyclic submodules. Recall that for an eigenvalue $a$ of $g, V((a))$ is the submodule consisting of all $v$ with $(g-a)^{k}(v)=0$, for large $k$.

Proposition 3.1. Let $g \in \mathcal{O}(V, B)$. Then: (1) $V((a))$ and $V((b))$ are orthogonal, unless $a b=1$.

(2) The cyclic decompositions of $V((1))$ and $V((-1))$ can be chosen so that cyclic pieces of different dimensions are orthogonal.

Proof. This is a special case of Lemma 3.1 and Theorem 3.2 of [2].

Let $g \in \operatorname{End}(V)$. For each eigenvalue $a_{\imath}$ of $g$, let $n_{1}=\operatorname{dim} V\left(\left(a_{1}\right)\right)$ and let $P_{a}$ be the partition of $n_{t}$ given by the degrees of the elementary divisors of $g$ corresponding to $a_{t}$.

Proposition 3.2. (1) Suppose $g \in \mathcal{O}(V, B)$ and $a_{1}= \pm 1, P_{t}=$ $\left(m_{1}, \cdots, m_{k}\right)$. If an even number occurs among the $m_{t}$, it must occur an even number of times.

(2) Suppose $g \in \mathcal{O}(V, B)$ and $a_{1} \neq \pm 1$. Then, for some $j, a_{\imath}^{-1}=a_{l}$, $n_{t}=n_{1}$, and the partitions $P_{1}$ and $P_{1}$ are the same.

(3) If $g \in \operatorname{End}(V)$ has Jordan form satisfying the conditions in (1) and (2), then there exists a nondegenerate symmetric bilinear form $B$ on $V$ with $g \in \mathcal{O}(V, B)$.

Proof. This is all a special case of Theorem 3.4 of [2] and his discussion on pp. 94-95, 97.

The proofs of Theorem (1.2) and Proposition (1.3) are now easily done. If $g \in \mathscr{O}(V, B)$ and $\mathscr{D}(g) \geqq \frac{1}{2}(n-2)(n-3)$, then the possible partitions induced by the Jordan form of $g$ are known, by Propositions (2.5) and (3.2). Eigenvalues can then be assigned to these partitions, subject to the restrictions in Proposition (3.2). Then, the possible Jordan forms for $g$ are known, and Proposition (3.1) tells which pieces may be taken to be orthogonal. We omit the details.

\section{REFERENCES}

1. E. P. Botta and S. Pierce, The preservers of any orthogonal group, Pacific J. Math., (to appear).

2. J. Milnor, On isometries of inner product spaces, Invent. Math., 8 (1969), 83-97.

Received March 20, 1977. Partially supported by an NSF grant. 


\section{PACIFIC JOURNAL OF MATHEMATICS}

\section{EDITORS}

RICHARD ARENS (Managing Editor)

University of California

Los Angeles, CA 90024

R. A. BeAumont

University of Washington

Seattle, WA 98105

C. C. MOORE

University of California

Berkeley, CA 94720
J. DUGUNDJI

Department of Mathematics

University of Southern California

Los Angeles, CA 90007

R. FINN AND J. MILGRAM

Stanford University

Stanford, CA 94305

\section{ASSOCIATE EDITORS}
E. F. BECKENBACH
B. H. NEUMANN
F. WOLF
K. YoshidA

\section{SUPPORTING INSTITUTIONS}

UNIVERSITY OF BRITISH COLUMBIA

UNIVERSITY OF SOUTHERN CALIFORNIA

CALIFORNIA INSTITUTE OF TECHNOLOGY

STANFORD UNIVERSITY

UNIVERSITY OF CALIFORNIA

UNIVERSITY OF HAWAII

MONTANA STATE UNIVERSITY

UNIVERSITY OF TOKYO

UNIVERSITY OF NEVADA

UNIVERSITY OF UTAH

NEW MEXICO STATE UNIVERSITY

OREGON STATE UNIVERSITY

UNIVERSITY OF OREGON

OSAKA UNIVERSITY

WASHINGTON STATE UNIVERSITY

UNIVERSITY OF WASHINGTON

AMERICAN MATHEMATICAL SOCIETY

The Supporting Institutions listed above contribute to the cost of publication of this Journal, but they are not owners or publishers and have no responsibility for its contents or policies.

Mathematical papers intended for publication in the Pacific Journal of Mathematics should be in typed form or offset-reproduced (not dittoed), double spaced with large margins. Underline Greek letters in red, German in green, and script in blue. The first paragraph or two must be capable of being used separately as a synopsis of the entire paper. Items of the bibliography should not be cited there unless absolutely necessary, in which case they must be identified by author and Journal, rather than by item number. Manuscripts, in duplicate, may be sent to any one of the four editors. Please classify according to the scheme of Math. Reviews, Index to Vol. 39. All other communications should be addressed to the managing editor, or Elaine Barth, University of California, Los Angeles, California, 90024.

100 reprints are provided free for each article, only if page charges have been substantially paid. Additional copies may be obtained at cost in multiples of 50 .

The Pacific Journal of Mathematics is issued monthly as of January 1966. Regular subscription rate: $\$ 72.00$ a year (6 Vols., 12 issues). Special rate: $\$ 36.00$ a year to individual members of supporting institutions.

Subscriptions, orders for numbers issued in the last three calendar years, and changes of address should be sent to Pacific Journal of Mathematics, 103 Highland Boulevard, Berkeley, California, 94708.

PUBLISHED BY PACIFIC JOURNAL OF MATHEMATICS, A NON-PROFIT CORPORATION

Printed at Jerusalem Academic Press, POB 2390, Jerusalem, Israel. 


\section{Pacific Journal of Mathematics}

\section{Vol. 75, No. $2 \quad$ October, 1978}

Susan Jane Zimmerman Andima and W. J. Thron, Order-induced

topological properties ................................... 297

Gregory Wade Bell, Cohomology of degree 1 and 2 of the Suzuki groups . . 319

Richard Body and Roy Rene Douglas, Rational homotopy and unique

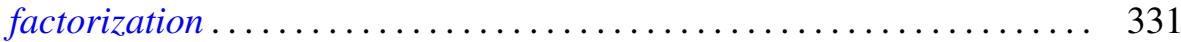

Frank Lewis Capobianco, Fixed sets of involutions ................. 339

L. Carlitz, Some theorems on generalized Dedekind-Rademacher sums .... 347

Mary Rodriguez Embry and Alan Leslie Lambert, The structure of a special class of weighted translation semigroups .....................

Steve Ferry, Strongly regular mappings with compact ANR fibers are

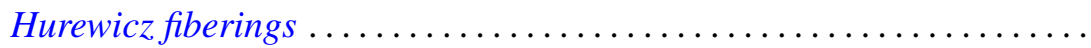

Ivan Filippenko and Marvin David Marcus, On the unitary invariance of the

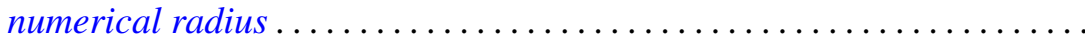

$\mathrm{H}$. Groemer, On the extension of additive functionals on classes of convex

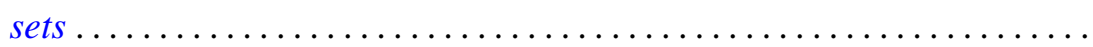

Rita Hall, On the cohomology of Kuga's fiber variety ............... 411

H. B. Hamilton, Congruences on $\mathrm{N}$-semigroups ................. 423

Manfred Herrmann and Rolf Schmidt, Regular sequences and lifting

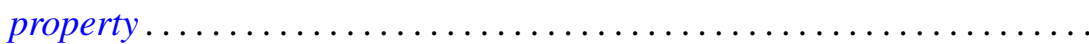

James Edgar Keesling, Decompositions of the Stone-Čech compactification which are shape equivalences .....................

Michael Jay Klass and Lawrence Edward Myers, On stopping rules and the expected supremum of $S_{n} / T_{n}$

Ronald Charles Linton, $\lambda$-large subgroups of $C_{\lambda}$-groups

William Owen Murray, IV and L. Bruce Treybig, Triangulations with the free cell property ............................

Louis Jackson Ratliff, Jr., Polynomial rings and $H_{i}$-local rings ...

Michael Rich, On alternate rings and their attached Jordan rings....

Gary Sampson and H. Tuy, Fourier transforms and their Lipschitz

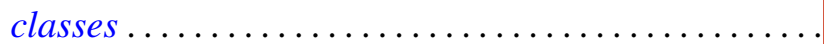

Helga Schirmer, Effluent and noneffluent fixed points on dendrites ...

Daniel Byron Shapiro, Intersections of the space of skew-symmetric maps

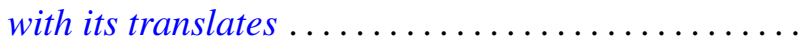

Edwin Spanier, Tautness for Alexander-Spanier cohomology ...

Alan Stein and Ivan Ernest Stux, A mean value theorem for binary digits ...

Franklin D. Tall, Normal subspaces of the density topology . .

William Yslas Vélez, Prime ideal decomposition in $F\left(\mu^{1 / p}\right) \ldots$ 